\title{
Cutting Edge in Medical Management of Cutaneous Oncology
}

\author{
Kim Chong, MD, ${ }^{*}$ Adil Daud, MD, ${ }^{\dagger}$ Susana Ortiz-Urda, MD, PhD, ${ }^{*}$ and \\ Sarah T. Arron, MD, PhD*; for the UCSF High Risk Skin Cancer Program
}

\begin{abstract}
Traditional chemotherapy has resulted in only a modest response, if any, for the 3 most common cutaneous malignancies of basal cell carcinoma, squamous cell carcinoma, and melanoma. Recent advances in understanding of the defects in the pathways driving tumorigenesis have changed the way that we think of these cancers and paved the way to targeted therapy for specific tumors. In this review, we will introduce the novel systemic treatments currently available for these cancers in the context of what is understood about the tumor pathogenesis. We will also introduce ongoing studies that will hopefully broaden our options for highly effective and tolerable treatment.

Semin Cutan Med Surg 31:140-149 (C) 2012 Elsevier Inc. All rights reserved.
\end{abstract}

KEYWORDS melanoma, basal cell carcinoma, squamous cell carcinoma, chemotherapy, pathway inhibitor, immunotherapy

$\mathrm{T}$ he objective of this review is to discuss the novel systemic treatments available for the management of metastatic basal cell carcinoma (BCC), squamous cell carcinoma (SCC), and melanoma. Although surgical excision is the gold standard treatment for all of these cutaneous malignancies, extensive locally destructive or metastatic disease still poses a therapeutic challenge, and treatments are rarely curative. Traditional treatment is highly toxic and the nonspecificity of the mechanism of action makes it impossible to determine who will respond to treatment. The advent of a molecular targeted therapy is changing the therapeutic landscape for

*Department of Dermatology, University of California at San Francisco, San Francisco, CA.

$\uparrow$ Division of Hematology and Oncology, Department of Medicine, University of California at San Francisco, San Francisco, CA.

Conflicts of Interest Disclosures: All authors have completed and submitted the ICMJE Form for Disclosure of Potential Conflicts of Interest. The institution of Dr. Daud has received funds for grants from Merck, Pfizer, GlaxoSmithKline, Sherry, and OncoSec; consulting fee or honorarium from Merck and BMS; fees for participation in review activities from Scherring, Merck, and Bristol-Myers Squibb; has grants/grants pending with Merck, Scherring, Bristol-Myers Squibb, Pfizer, and Oncosec; has received funds for services provided to speakers bureaus from Scherring, Bristol-Myers Squibb, and Merck. Dr. Daud has personally received support for travel to meetings from Scherring. All other authors have no conflicts to report.

Address reprint requests to Sarah T. Arron, MD, PhD, Department of Dermatology, University of California at San Francisco, Box 0316, 1701 Divisadero St, San Francisco, CA 94143.E-mail: arrons@derm.ucsf.edu these diseases, with an increased therapeutic index and, in many cases, with a more tolerable toxicity profile.

\section{Basal Cell Carcinoma}

BCC is the most common form of skin cancer, with an incidence rate that is 4-5 times more than SCC. It is typically slow growing, but if left untreated, local invasion may occur, leading to destruction, disfigurement, and rarely metastasis. The options available for treatment of the local disease include surgery, destruction, radiation, topical immunomodulation, and topical chemotherapy. Locally advanced BCC may invade underlying muscle, bone, or other contiguous structures. Metastatic disease is rare, but can be life threatening. A systemic therapy is warranted in cases in which local modalities are insufficient. There have been variable successes with cisplatinum-based chemotherapy regimens in the past. ${ }^{1}$

Recent advances in the understanding of the pathogenesis of BCC have led to the development of therapeutics that target the biological mechanism driving this malignancy. The Hedgehog (Hh) pathway has been shown to play a key role in the pathogenesis of BCC, with most BCC bearing mutations in genes in this developmental pathway. Most mutations implicated in BCC pathogenesis involve mutations in the transmembrane proteins, with loss of function of the patched homologue 1 (Ptch1) or gain of function of the smoothened homolog (Smo). ${ }^{2}$ Mutation in the Ptchl gene was initially 


\section{Hedgehog pathway}

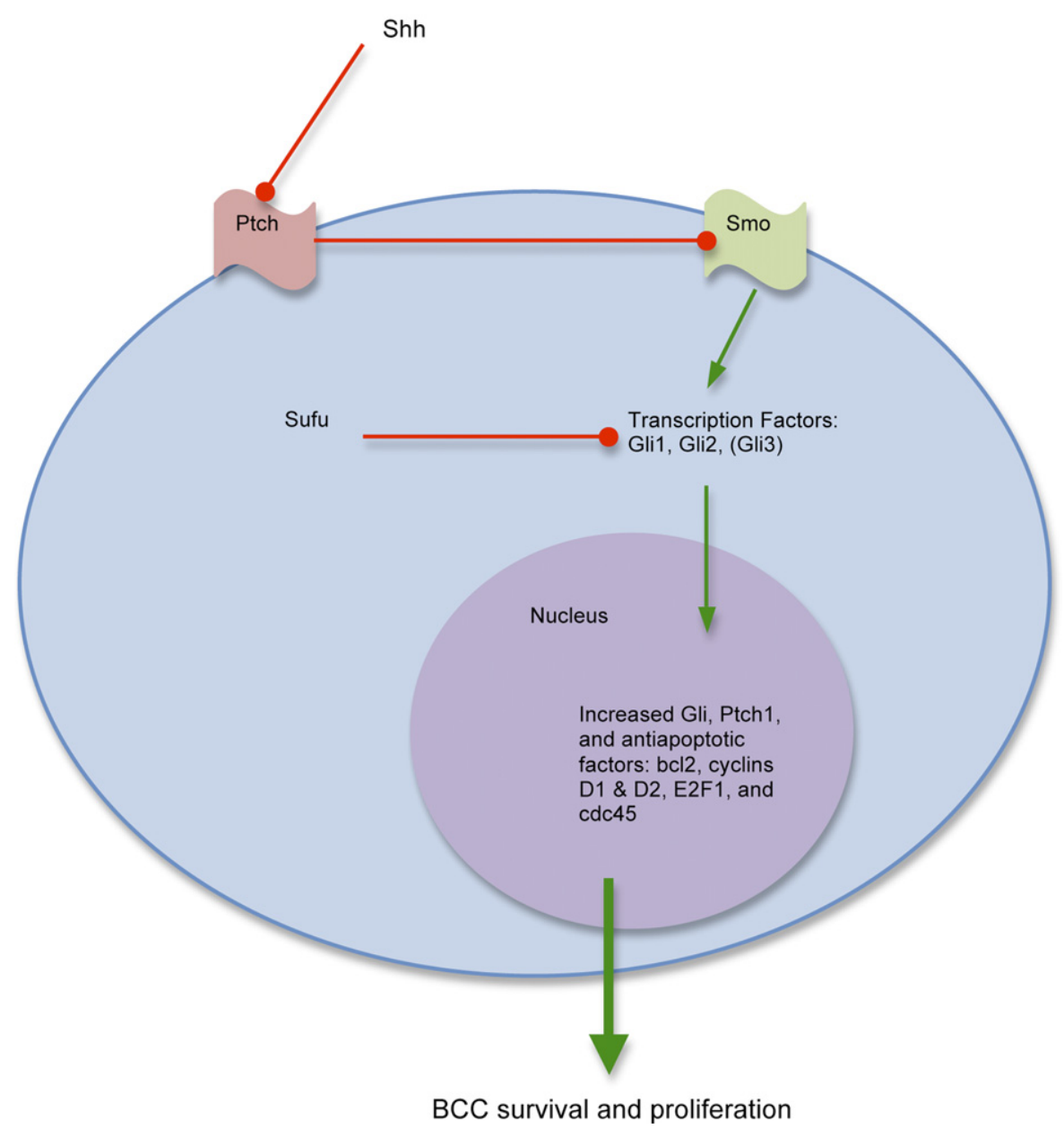

Figure 1 Schematic of the Hedgehog pathway involved in basal cell carcinoma tumorigenesis.

implicated as the cause of the rare autosomal dominant heritable basal cell nevus syndrome (Gorlin syndrome), the hallmark of which is a high susceptibility rate for the development of BCCs. ${ }^{3,4}$ It was later found that essentially all BCCs harbored mutation in the Ptch1 gene or other alterations in the Hh signaling pathway. ${ }^{5}$

The Ptchl is a keratinocyte membrane protein that binds Sonic Hh. In the absence of Sonic Hh, the role of the normal Ptchl is to inhibit Smo. Smo enables the activation of a family of transcription factors called Gli, which then enters the nucleus to promote expression of more Gli, Ptchl, and other apoptotic factors, and suppression of genes associated with keratinocyte differentiation. This sequence of events leads to cell proliferation and increased survival.

Overexpression of Ptchl, Smo, Gli1, and Gli2 is associated with BCC. ${ }^{6}$ This makes the Hh pathway both an attractive and a logical target for molecular inhibitors for treatment of BCC. Several hedgehog pathway inhibitors (HPIs) are under development in both oral and topical formulations. Currently, all are HPI against Smo (Fig. 1).

Vismodegib (Genentech/Roche), previously known as RG3616 or GDC-0449, is the first of the oral small molecule HPI against Smo to be approved by the FDA for locally ad- vanced or metastatic BCC. In phase I testing, 18 of 33 patients with locally advanced or metastatic BCC showed a response to the drug, and 11 treated patients had stable disease (SD) during a median follow-up of 9.8 months. Measurement of Gli in treated tumors was lower, demonstrating a downregulation of the Hh pathway, thus confirming the molecular mechanism of action. ${ }^{7}$ In a phase II trial, looking at locally advanced BCC not amenable for surgery or radiation and metastatic BCC, the overall response rate was $43 \%$ in the locally advanced group and $30 \%$ in the metastatic group. The median duration of progression-free survival (PFS) for both groups was 9.5 months. ${ }^{8}$

LDE225 (Novartis) is another oral HPI targeting Smo. In a phase I dose-escalation study in solid tumors, LDE225 was found to have a dose-dependent inhibition of the Hh pathway, which was measured by the downregulation of Glil expression. Although the trial was not designed to test for efficacy, it is notable that only 1 of 7 subjects with BCC progressed while on treatment. ${ }^{9}$ Phase II trials are under way. Other systemic HPIs are in development. These include IPI926 (Infinity Pharmaceuticals), TAK-441 (Millennium Pharmaceuticals), PF-04449913 (Pfizer), LEQ506 (Novartis), and BMS-833923 (Bristol-Myers Squibb). 
Topical delivery of HPIs is also under investigation and provides an attractive option in terms of side effect profile. CUR61414 (Curis/Genentech/Roche) was shown to be effective in preclinical models, but failed to have clinical activity in superficial or nodular BCCs in a phase I clinical study in humans. ${ }^{10} \mathrm{LDE} 225$ was also formulated as a topical cream. In a randomized, vehicle-controlled, intraindividual trial in subjects with basal cell nevus syndrome, topical LDE225 resulted in clinical responses in 12 of 13 BCCs studied, whereas tumors treated with vehicle alone showed no efficacy. ${ }^{11}$ Although these are promising results, further studies will be needed to test whether the findings are generalizable for patients with BCC without the syndrome.

Locally advanced and metastatic BCC portends a grave prognosis. The standard of care remains the cisplatin-based chemotherapy. However, the landscape of treatment for BCC is on the cusp of changing with the advent of HPIs and targeted molecular approach to treatment, and it is hoped that the prognosis for advanced disease will improve as well.

\section{Melanoma}

Melanoma is a devastating disease once metastatic and is the leading cause of skin cancer death. ${ }^{12}$ Systemic treatment for metastatic melanoma includes chemotherapy, immunotherapy, and more recently targeted therapy. Chemotherapy has been the standard of care for stage IV unresectable melanoma, with only a modest response and no improvement in overall survival (OS). ${ }^{13}$ With the advent of targeted therapy, we now understand melanoma to be a heterogeneous entity, with responses to treatment dependent on genetic status. Therefore, management of systemic disease now necessitates obtaining genetic analysis before a discussion of the options available for an individual patient.

\section{Immunotherapy}

Immunotherapy has long been known to play an important role in controlling melanoma and has been used in the adjuvant setting. Interferon $\alpha$-2b and, more recently, granulocyte macrophage colony-stimulating factor have been shown to improve PFS and OS in high-risk melanoma in the adjuvant setting. For metastatic melanoma, immune therapy options include interleukin-2, ipilimumab, and interleukin-12.

High-dose interleukin-2 can be highly efficacious in a limited subset of patients, with an overall response rate of $16 \%$ and a complete response rate of $6 \%-8 \% .{ }^{14}$ However, it causes significant toxicities and adverse events, such as hypotension, renal insufficiency, hepatocellular damage, edema, respiratory compromise, myocardial infarction, sepsis, and death. Given the side effect profile, it is often a treatment reserved only for young and fit patients. ${ }^{15}$ There are currently no biomarkers to determine who could likely benefit from the treatment; however, it has been found that patients with disease limited to subcutaneous tissue and those who are able to receive more dosages have been more likely to achieve an objective response. ${ }^{16}$
Ipilimumab (Bristol-Myers Squibb) is a CTLA-4 antibody and has been recently approved by the FDA for the treatment of metastatic melanoma. CTLA-4 competes for binding of a surface protein B7, thus inhibiting T-cell proliferation and release of immune stimulatory cytokines. Ipilimumab blocks CTLA-4, thus taking the proverbial brakes off the immune system and allowing the immune system to act against melanoma. ${ }^{17}$ In a phase 3 study comparing ipilimumab, with or without glycoprotein 100 (gp100) peptide vaccine, with gp100 alone in patients with previously treated melanoma, there was a statistically significant improved survival rate associated with treatment with ipilimumab. Median OS in the ipilimumab groups was not different and was about 10 months compared with 6.1 months for the gp100-alone group. A major drawback of this treatment has been the lag in treatment response. ${ }^{18}$

IL-12 is a heterodimeric cytokine that regulates both innate and adaptive immune response..$^{19,20}$ It has been shown to enhance the killing of tumor cells by tumor-infiltrating lymphocytes in patients with melanoma. ${ }^{21,22}$ Local delivery of IL-12 via direct intratumoral injection of IL-12 plasmid DNA is well tolerated and has been shown to result in local effects in the treated tumor but no systemic effect. ${ }^{23}$ Phase I and II trials of systemic IL-12 have been reported with responses in melanoma but are associated with significant toxicity. ${ }^{24-26}$ More recently, a phase I study of intratumoral electroporation of a DNA plasmid expressing IL-12 into melanoma lesions has been shown to result in regression of untreated metastases in 10 of 19 evaluable patients and is not associated with significant side effects. ${ }^{27}$ Future studies are proposed.

Although immunotherapy has been shown to make an impact in melanoma beyond just the adjuvant setting, it is still poorly understood why subsets of patients show a better response than others. Further investigations are needed to determine why responders respond and possibly what can be done to convert a nonresponder to a responder. Because targeted therapies become the standard of care in melanoma, immunotherapy has become second-line treatment reserved for patients who did not respond to targeted treatment or for those who do not qualify for targeted treatment. Studies are under way combining immunotherapy with targeted therapy such as vemurafenib, a BRAF inhibitor, to potentially boost the efficacy of both.

\section{Therapies Targeting Molecular Signaling}

There are 2 main pathways currently recognized to play a role specifically in melanoma pathogenesis. These are the mitogen-activated protein kinase (MAPK) pathway and the phosphatidylinositol 3-kinase (PI3K) pathway. Recognizing these pathways and developing drugs that target specific points that are dysregulated in the pathways have several advantages. The specificity allows for targeted treatment with fewer side effects. Additionally, because responders are chosen based on their genetic status, it becomes possible to predict clinical response without subjecting nonresponders to treatment that will not be effective for them. Table 1 is a 
Table 1 Systemic Therapy for Melanoma

\begin{tabular}{|c|c|c|}
\hline Class & Medication & Mechanism of Action \\
\hline \multirow[t]{3}{*}{ Immunotherapy } & High-dose IL-2 & $\begin{array}{l}\text { IL-12 mediated killing of tumor via tumor- } \\
\text { infiltrating lymphocytes }\end{array}$ \\
\hline & Ipilimumab & CTLA-4 antibody \\
\hline & Intratumoral electroporation of IL-12 & $\begin{array}{l}\text { Gene transfer using in vivo DNA electroporation } \\
\text { of IL-12 leading to IL-12 mediated killing of } \\
\text { tumor }\end{array}$ \\
\hline \multirow[t]{23}{*}{ Targeted therapy } & Vemurafenib & BRAF inhibitor \\
\hline & GSK118436 & BRAF inhibitor \\
\hline & Sorafenib & Nonselective RAF inhibitor \\
\hline & Selumetinib (AZD6244) & MEK inhibitor \\
\hline & GSK1120212 & MEK inhibitor \\
\hline & MEK162 & MEK inhibitor \\
\hline & Sirolimus & mTOR inhibitor \\
\hline & Temsirolimus (CCL-779) & mTOR and VEGF inhibitor \\
\hline & Everolimus & mTOR and VEGF inhibitor \\
\hline & BEZ235 & mTOR and PI3K inhibitor \\
\hline & Cediranib & VEGF inhibitor \\
\hline & Perfosine & AKT inhibitor \\
\hline & UCN-01 & PDK-1 inhibitor \\
\hline & Imatinib & Kit inhibitor \\
\hline & Nilotinib & Kit inhibitor \\
\hline & Sunitinib & Kit inhibitor \\
\hline & Dasatinib & Kit inhibitor \\
\hline & Masitinib & Kit inhibitor \\
\hline & Cabozantinib (XL184) & c-MET and VEGFR2 inhibitor \\
\hline & Foretinib (XL880) & c-MET and VEGFR2 inhibitor \\
\hline & Gefitinib & ErbB1/B2 inhibitor \\
\hline & Bevacizumab & EGFR inhibitor \\
\hline & Ranibizumab & Monoclonal antibody fragment VEGF-A inhibitor \\
\hline
\end{tabular}

summary of the targeted therapies mostly under investigation in melanoma, with the exception of vemurafenib and ipilimumab, which have been FDA-approved.

\section{MAPK Signaling Pathway}

MAPK signaling is initiated by binding of receptor tyrosine kinases, which then lead to activation of Ras, a small G protein on the inner surface of the cell membrane. Once activated, Ras can form complexes with Raf, which then leads to phosphorylation of extracellular signal-regulated kinase (ERK) via activation of mitogen-activated ERK kinase (MEK). ERK can directly enter the nucleus and effect translation of genes and control cellular proliferation (Fig. 2). ${ }^{28}$

\section{Serine/Threonine-Protein Kinase B-Raf}

Mutation in the BRAF gene occurs in approximately $66 \%$ of melanoma tumors and is commonly found in nonchronically sun-exposed skin. ${ }^{29}$ The BRAF mutation is uncommon in acral lentiginous melanoma, but we have observed several cases of it at our institution. The BRAF mutation confers increased kinase activity that can lead to increased tumor proliferation. ${ }^{30}$ Inhibition of the mutated BRAF gene has been shown to be the most effective treatment for melanoma at this time and should be considered first-line treatment for melanoma bearing this mutation. In a recently published phase III trial of oral vemurafenib (Genentech/Roche), a new recently FDA-approved BRAF-selective inhibitor for metastatic pa- tients bearing the BRAF V600E mutation showed a $48 \%$ response rate for vemurafenib compared with a $5 \%$ response rate for dacarbazine. A 6-month interim analysis showed a $63 \%$ reduction in risk of death and a $74 \%$ reduction in risk of death and disease progression in the vemurafenib versus the dacarbazine group ( $P<.0001$ for both comparisons). Another favorable aspect of treatment with vemurafenib is its relatively benign side effect profile. ${ }^{31}$ Common side effects of vemurafenib include rash, fatigue, arthralgia, alopecia, photosensitivity, nausea, diarrhea, keratoacanthoma or SCC, and, to a lesser extent, liver function abnormalities and renal insufficiency.

The recent FDA approval of vemurafenib has changed the landscape for management of metastatic melanoma and has caused significant excitement in the melanoma community. Unfortunately, what is not clearly understood is why there is variable response to the medication despite the presence of the BRAF mutation, implying that other important factors play a role in melanoma tumorigenesis. Also, among the initial responders, most patients eventually progress on treatment. Some mechanisms of resistance have been proposed but have thus far not been validated. ${ }^{32}$

\section{Mitogen-activated ERK Kinase}

MEK is a downstream target of Raf in the signaling cascade. It has been shown to have mixed results in melanoma. A phase 
MAPK and PI3K/AKT pathway

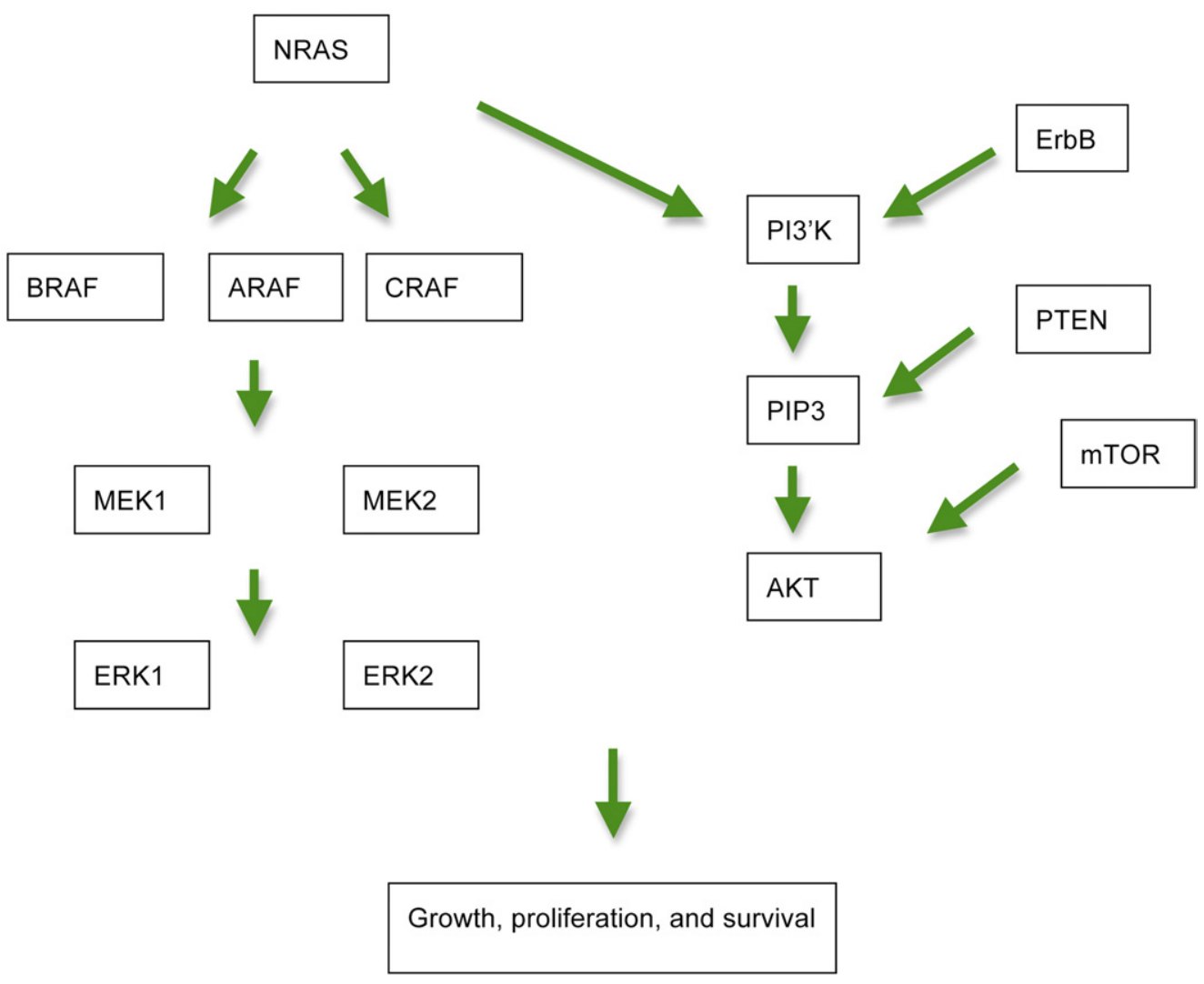

Figure 2 Simplified schematic of MAPK and PI3K/AKT mediated pathway in melanoma tumorigenesis.

I trial with AZD6244 (AstraZeneca) showed tumor shrinkage in 6 of 11 patients. ${ }^{33}$ However, a subsequent phase II trial showed partial response in some patients, mostly in those with the BRAF mutations, but there was no benefit in PFS when compared with temozolomide, which is an oral alkylating chemotherapy commonly used in melanoma. ${ }^{34}$ MEK in combination with temozolomide, docetaxel, or temsirolimus has been shown to be associated with tumor regression in only BRAF mutants and delayed progression in BRAF and NRAS mutants. ${ }^{35}$ This suggests that MEK plays possibly a bigger role in tumorigenesis in BRAF mutants than in NRAS mutants. Studies are currently under way comparing AZD6244 in combination with dacarbazine (in BRAF mutant melanoma only $)^{36}$ or docetaxel versus chemotherapy alone. ${ }^{37}$ Two other studies hope to compound the MEK-inhibiting effects of AZD6244 by targeting parallel growth pathways with a mammalian target of rapamycin (mTOR) and vascular endothelial growth factor receptor (VEGF) inhibitor temsirolimus (BRAF mutant melanoma only) ${ }^{38}$ or a VEGF inhibitor cediranib. ${ }^{39}$ A newer MEK inhibitor GSK1 120212 is currently under investigation in a phase I trial in combination with a BRAF inhibitor GSK2118436 for metastatic BRAF mutant melanoma. ${ }^{40}$ It is showing great promise, indicating possibly that dual targets in the same pathway are more effective than a single one.

\section{PI3K/AKT Pathway}

The PI3K pathway is a prosurvival pathway, antagonizing apoptosis. PI3K is activated by growth factor receptors. It has 2 actions: to regulate cell proliferation via control of entry into the cell cycle and to activate AKT via PDK1. AKT then directly activates transcription factors that cause transcription of prosurvival genes. The PI3K/AKT pathway is constitutively activated in melanoma, although mutations in AKT are found in only a small proportion of melanomas. ${ }^{41} \mathrm{mTOR}$ is a serine/threonine kinase downstream in this pathway that leads to increased cell growth. Increased activation of mTOR was found in $73 \%$ of melanoma cell lines. ${ }^{42}$ This pathway can be opposed by PTEN. ${ }^{43}$ Mutation in PTEN has been found in $11 \%$ of melanoma tumors ${ }^{44}$ and $43 \%$ of melanoma cell lines. ${ }^{45}$ Several inhibitors of this pathway are in the early stages of development, all targeting this pathway from different angles. Thus far, none of the inhibitors of this pathway have demonstrated an objective response (Fig. 2).

Perifosine (Aeterna Zentaris) is an AKT inhibitor, inhibiting AKT phosphorylation and translocation to the cell membrane. Unfortunately, a phase II trial using this drug in metastatic melanoma showed only stabilization of disease in 3 of 14 patients and was associated with side effects requiring missed, delayed, or reduced dose in all patients. ${ }^{46}$ 
UCN-01 (Kyowa Hakko Kogyo) is an inhibitor of PDK-1, which activates AKT, thus leading to decreased apoptosis. A phase I trial demonstrated PR in one patient on this medication. A subsequent phase II trial of UCN-O1 in metastatic melanoma accrued 16 evaluable patients, with 4 patients demonstrating SD and 12 progressive disease. Median PFS was 1.3 months, and median OS was 7.3 months. It was relatively well tolerated. ${ }^{47}$

Various mTOR inhibitors are also being evaluated in patients with melanoma. Temsirolimus, CCL-779 (Wyeth), is an mTOR inhibitor that has been tested in a melanoma phase II clinical trial with disappointing results, with only $1 \mathrm{PR}$ lasting just 2 months. ${ }^{48}$ Everolimus (Abbott) is another mTOR inhibitor with dual activity against epidermal growth factor receptor (EGFR). It is currently under investigation in a phase II trial, which has thus far demonstrated 7 of 24 patients with stabilization of disease in an interim analysis. ${ }^{49}$

\section{Dual Pathway Inhibition}

It is believed that both the MAPK and the PI3K/AKT pathways play key roles in melanoma tumorigenesis. Inhibition of the PI3K/AKT pathway alone has been disappointing without objective response. By contrast, Raf inhibition is limited to those bearing the BRAF mutation and is not durable in most cases. There is hope that using a combinatorial approach with inhibitors in both pathways will have an additive positive effect. In cell culture, the combination of sorafenib, a nonselective Raf inhibitor, with sirolimus, an mTOR inhibitor, caused a 2 -fold increase in apoptosis of melanoma cells relative to sorafenib alone. This was attributable to an upregulation in genes associated with endoplasmic reticulum stressinduced apoptosis. ${ }^{50}$ BEZ235 (Novartis) is another molecule with dual mTOR and PI3K inhibition that has shown greater activity than temsirolimus in preclinical melanoma models. ${ }^{51}$ There is also currently a phase Ib study under way, combining BEZ235 with the MEK inhibitor MEK162.52

\section{Kit}

Kit is a cytokine receptor that belongs to the type III receptor tyrosine kinase family. Kit signaling plays an important role in several physiological processes including melanogenesis. ${ }^{53}$ Overall, this mutation is rare but is most commonly found in melanoma located on chronically sun-damaged skin, mucosa, and acral skin. ${ }^{54}$ The overall incidence rate of Kit mutant melanoma has been reported to be $8 \% .{ }^{55}$

Imatinib (Novartis) was the earliest Kit inhibitor tested in clinical trials for melanoma. Two previous trials in which imatinib was tested for efficacy against melanoma demonstrated no treatment response. ${ }^{56,57}$ However, it is important to note that the patients were not tested for Kit mutation, and it was determined that most selected patients had tumors that demonstrated little to no Kit expression by immunohistochemistry. A recent phase II open-label, single-arm trial using imatinib only in Kit mutant metastatic melanoma recruited 43 patients; 23 patients showed complete response, 13 PR, and 10 SD. The median PFS was 3.5 months. ${ }^{58}$ Based on this study, it can be concluded that Kit inhibitors can play an important role in the armamentarium against selected melanoma bearing this mutation. Multiple trials are ongoing using newer tyrosine kinase inhibitors, such as nilotinib, sunitinib, dasatinib, and masitinib, against Kit-mutated melanoma.

\section{c-Met}

c-Met is a receptor tyrosine kinase that is activated by its ligand hepatocyte growth factor and is essential for normal development, cell migration, growth, survival, differentiation, and angiogenesis. ${ }^{59}$ In normal skin, $c$-Met is expressed on epithelial cells and melanocytes, whereas hepatocyte growth factor is produced mainly by mesenchymal cells and interacts with c-Met in a paracrine manner. ${ }^{60} \mathrm{c}$-Met has been found to be expressed in $88 \%$ of melanomas, ${ }^{61}$ with overexpression correlating with the invasive growth of melanoma cells. Many melanomas also secrete hepatocyte growth factor, which can induce sustained activation of c-Met in an autocrine fashion. ${ }^{62}$ Cabozantinib, XL184 (Exelixis), is a c-Met and VEGFR2 inhibitor found in a phase II randomized discontinuation trial of patients with advanced solid tumors that demonstrated a 5\% objective response rate in melanoma soft tissue disease. However, $87 \%$ of patients with metastatic pancreatic, breast, and melanoma were shown to have a partial or complete response in their bony metastases. ${ }^{63}$ Cabozantinib is currently in a phase II trial among patients with various solid tumors, including melanoma. ${ }^{64}$ Foretinib, XL880 (GlaxoSmithKline), is another c-Met/VEGFR2 inhibitor that also has shown some objective response activity in patients with melanoma in a phase I trial. ${ }^{65}$

\section{Epidermal Growth Factor Receptor}

The EGFR is a member of a family of transmembrane protein kinase receptors, which consists of the 4 receptors: EGFR (HER1 or ErbB1), ErbB2 (HER2), ErbB3 (HER3), and ErbB4 (HER4). ${ }^{66}$ The EGFR gene resides on chromosome 7. Several different ligands activate these receptors, which then relay signals to the parallel MAPK and PI3K pathways leading to growth effects, angiogenesis, migration, and invasion. EGFR has been shown to play an important role in the growth and survival of many tumors including cutaneous malignancies. Anti-EGFR agents are monoclonal antibodies directed at the extracellular domain of the receptor and low-molecular weight adenosine triphosphate (ATP)-competitive inhibitors of the receptor's intracellular tyrosine kinase.

ErbB1 has been found to be expressed in up to $96 \%$ of primary melanomas and in $90 \%$ of metastatic tumors. ${ }^{67}$ There are gains in chromosome 7, where EGFR resides, in about 50\% of melanomas, and increased copy number of chromosome 7 has been associated with poor prognosis in some studies. ${ }^{68,69}$ ErbB3 is also frequently expressed in melanoma and has been associated with tumor progression and a worse prognosis. ${ }^{70-72}$ Evidence of the importance of EGFR signaling has been seen in melanoma cell lines ${ }^{70}$ as well as in animal models. ${ }^{73}$ A screen for somatic mutations in ErbB4 revealed that $19 \%$ of metastatic tumors harbored this mutation. ${ }^{74}$ 
The ErbB1 inhibitor erlotinib hydrochloride (Genentech) has been evaluated in a phase II trial of metastatic melanoma and showed no objective responses, but 4 of 14 patients showed SD. ${ }^{75}$ The ErbB1/B2 inhibitor lapatinib is currently under investigation for melanoma bearing the ErbB4 mutation after preclinical data have suggested its effectiveness in ErbB4 mutant melanoma. The ErbB1/B2 inhibitor gefitinib (AstraZeneca) was tested, and only 2 of 50 evaluable patients showed PR. ${ }^{76}$ A trial of erlotinib hydrochloride in combination with VEGF-A inhibitor bevacizumab (Genentech/ Roche) showed greater efficacy, with 2 of 23 patients having PR lasting $<6$ months and 5 patients having SD lasting $>6$ months. ${ }^{77}$ Toxic effects were greater with this combination, with 2 reported cases of myocardial infarction and bowel perforation.

\section{VEGF Receptor}

Angiogenesis plays a major role in tumor growth. Targeting the VEGF makes logical sense and has been shown to affect tumor growth by inhibition of angiogenesis. Although this is considered targeted therapy, it does not specifically target melanoma cells. Among the VEGF inhibitors, bevacizumab is perhaps the most extensively studied anti-VEGF antibody. It is under investigation in combination with immunotherapy, chemotherapy, and other targeted treatments in metastatic melanoma. It seems to have a synergistic effect when used in conjunction with other systemic modalities.

\section{Bevacizumab With Immunotherapy}

A phase II trial of bevacizumab and high-dose interferon $\alpha-2 b$, which has antiangiogenic properties via downregulation of basic fibroblast growth factor, in metastatic melanoma resulted in a median PFS of 4.8 months and OS of 17 months as compared with historical control of bevacizumab alone with PFS of 3 months and OS of 8.5 months. Six patients had PR and 5 patients had SD for $>24$ weeks. ${ }^{78}$ In a phase I trial of bevacizumab and ipilimumab for stage III or IV melanoma, of the 21 patients who were evaluable, there were 8 who had $\mathrm{PR}$, all of whom had durable responses $>6$ months, and 6 had SD. Post-treatment biopsies showed activated vessel endothelium with extensive T-cell trafficking, which was not seen in patients treated with ipilimumab alone. These results suggest a synergistic effect of VEGF and CTLA4 blockade. ${ }^{79}$

\section{Bevacizumab With Chemotherapy}

There have been many trials looking at bevacizumab used in conjunction with various chemotherapeutic regimens, which include temozolomide, nab-paclitaxel, carboplatin/paclitaxel, and dacarbazine. They have shown a modestly improved PFS and/or OS relative to previously reported survival for bevacizumab alone. .0-84 $^{8}$

\section{Bevacizumab With Other Targets}

It is still too early to say that bevacizumab has a synergistic effect when used in conjunction with other targeted treatment. In a phase II trial of bevacizumab and everolimus, median PFS was 4 months and OS was 8.6 months. ${ }^{85} \mathrm{~A}$ triple combination trial of temozolomide, sorafenib (Raf kinase inhibitor), and bevacizumab in 11 patients with refractory acral
Table 2 Systemic Therapy for Squamous Cell Carcinoma

\begin{tabular}{ll}
\hline Medication & \multicolumn{1}{c}{ Mechanism of Action } \\
\hline Cetuximab & EGFR monoclonal antibody \\
Panitumumab & IgG2 monoclonal antibody against \\
& EGFR \\
Matuzumab & Humanized IgG1 monoclonal antibody \\
& against EGFR \\
Gefitinib & EGFR TKI \\
Erlotinib & ErbB1 inhibitor \\
\hline
\end{tabular}

advanced melanoma resulted in 1 CR, 2 PR, and 6 SD. ${ }^{86}$ However, an interim report of another triple combination regimen of bevacizumab, oxaliplatin, and sorafenib in a phase I/II trial accruing 6 patients showed 1 PR, 3 mixed response, and 3 progression of disease. ${ }^{87}$ Additionally, there are trials ongoing combining dasatinib and bevacizumab.

\section{Ranibizumab}

Ranibizumab (Genentech) is a monoclonal antibody fragment derived from the same parent mouse antibody as bevacizumab. It is much smaller than the parent molecule and has been affinity matured to provide stronger binding to VEGF-A. There are currently multiple trials using this molecule for choroidal and uveal melanoma as an adjuvant for tumor control or for control of radiation retinopathy or maculopathy.

\section{Squamous Cell Carcinoma}

SCC has been shown to have an increased expression of EGFR, with about $92 \%-100 \%$ of SCC demonstrating binding to EGFR antibody. ${ }^{88,89}$ High EGFR signaling has been associated with aggressive disease, poor response to therapy, increased development of resistance to cytotoxic chemotherapy, poor survival, and poor prognosis. ${ }^{90}$ Another study found that primary SCC tumors were immunohistochemically focally weakly positive for EGFR, whereas metastatic SCCs were diffusely strongly positive, suggesting that stronger expression of EGFR had a higher potential for metastasis. ${ }^{91}$ Table 2 summarizes the systemic therapies available or under investigation for squamous cell carcinoma.

Cetuximab (Merck) is an anti-EGFR monoclonal antibody approved by the FDA for SCC of the head and neck. It binds with higher affinity than natural ligands TGF $\alpha$ and EGF. Cetuximab inhibits progression in the cell cycle at the G0/G1 phase, increases expression of the cell cycle regulator p27KIP1, and induces apoptosis by increasing expression of proapoptotic proteins or by inactivation of antiapoptotic proteins. ${ }^{92}$ It can also inhibit angiogenesis via inhibition of VEGF, interleukin-8, and basic fibroblast growth factor. ${ }^{93}$ Cetuximab has been shown to be effective in case reports for recurrent unresectable SCC as well as metastatic disease..$^{94-96}$ There have been several phase I and II trials using cetuximab in combination with platinum-based chemotherapy documenting safety and efficacy in the combination regimen in metastatic, recurrent, or refractory SCC of the head and neck. ${ }^{97-99}$ However, larger studies have not been conducted 
documenting effectiveness as a monotherapy. Predictive biomarkers for success with cetuximab include presence of EGFR in the tumor and wild-type for K-Ras ${ }^{100}$ and BRAF. ${ }^{101}$ The rationale is that these mutations constitutively activate the downstream MAPK pathway that is independent of EGFR activity.

Panitumumab, ABX-EGF (Amgen), is a human IgG2 monoclonal antibody against EGFR that binds to EGFR like cetuximab. Phase I trials have shown it to be well tolerated and efficacious in colorectal carcinoma and non-small cell lung cancer. An open-label phase II trial is currently under way to study the clinical efficacy in SCC. Matuzumab, EMD 72000 (Merck/Takeda), is a humanized IgGl monoclonal antibody against EGFR. It has been shown to have tumor response against esophageal SCC, cervical carcinoma, ovarian carcinoma, colorectal carcinoma, and head and neck SCC. ${ }^{102}$ A phase II trial of matuzumab in patients with platinum-resistant ovarian carcinoma showed that matuzumab was well tolerated and demonstrated evidence of antitumor activity. ${ }^{103}$ There are currently no studies at this time using this drug in the treatment of nonmelanoma skin cancer.

\section{EGFR TKI and Nonmelanoma Skin Cancer}

TKIs are synthetic low molecular weight molecules that interact with the intracellular tyrosine kinase domain of several receptors, including EGFR. They inhibit ligand-induced receptor phosphorylation by competing for intracellular $\mathrm{Mg}$ adenosine triphosphate-binding sites. ${ }^{104}$ Gefitinib has been shown to inhibit EGFR and MAPK activation and Pak 1 activity in exponentially growing cutaneous SCC. It has been approved for the treatment of nonsquamous cell lung cancer after platinum-based or docetaxel-based therapy failure and has been shown to have modest activity in advanced skin SCC. ${ }^{105}$ Erlotinib is a potent reversible, selective inhibitor of EGFR (ErbB1), ${ }^{106}$ which is also approved for nonsquamous cell lung cancer but has demonstrated effectiveness in other cancers as well. ${ }^{107}$ There are several studies under way to investigate its utility in cutaneous SCC in combination with radiotherapy, as an adjuvant or as a neoadjuvant therapy.

\section{Conclusion}

Management of cutaneous malignancy has entered a new era. Understanding the molecular basis for tumorigenesis has paved the way toward the development of new molecules that inhibit at critical sites necessary for neoplastic growth and survival. Some agents have shown startling efficacy and yet others have equally surprised with their lack of efficacy, making the poignant message that although the frontier of our understanding has advanced significantly, the entire story is yet to be told.

\section{References}

1. Pfeiffer P, Hansen O, Rose C: Systemic cytotoxic therapy of basal cell carcinoma. A review of the literature. Eur J Cancer 26:73-77, 1990

2. Epstein EH: Basal cell carcinomas: Attack of the hedgehog. Nat Rev Cancer 8:743-754, 2008

3. Hahn H, Wicking C, Zaphiropoulous PG, et al: Mutations of the human homolog of Drosophila patched in the nevoid basal cell carcinoma syndrome. Cell 85:841-851, 1996

4. Johnson RL, Rothman AL, Xie J, et al: Human homolog of patched, a candidate gene for the basal cell nevus syndrome. Science 272:16681671,1996

5. Xie J, Murone M, Luoh SM, et al: Activating smoothened mutations in sporadic basal-cell carcinoma. Nature 391:90-92, 1998

6. Gupta S, Takebe N, Lorusso P, et al: Targeting the hedgehog pathway in cancer. Ther Adv Med Oncol 2:237-250, 2010

7. Von Hoff DD, LoRusso PM, Rudin CM, et al: Inhibition of the hedgehog pathway in advanced basal-cell carcinoma. N Engl J Med 361: 1164-1172, 2009

8. Sekulic A, Migden MR, Oro AE, et al: A pivotal study evaluating efficacy and safety of the hedgehog pathway inhibitor (HPI) vismodegib (GDC-0449) in patients with locally advanced or metastatic basal cell carcinoma (BCC). Presented at the 7th Congress of the European Association of Dermato-Oncology, 2011, Nantes, France

9. Tawbi HA, Rodon Ahnert J, Dummer R, et al: Phase I study of LDE225 in advanced solid tumors: Updated analysis of safety, preliminary efficacy, and pharmacokinetic-pharmacodynamic correlation. J Clin Oncol 29, 2011. Abstract 3062

10. Tang T, Tang JY, Li D, et al: Targeting superficial or nodular basal cell carcinoma with topically formulated small molecule inhibitor of smoothened. Clin Cancer Res 17:3378-3387, 2011

11. Skvara H, Kalthoff F, Meingassner JG, et al: Topical treatment of basal cell carcinomas in nevoid basal cell carcinoma syndrome with a smoothened inhibitor. J Invest Dermatol 131:1735-1744, 2011

12. Jemal A, Siegel R, Ward E, et al: Cancer statistics. CA Cancer J Clin 2007:43-66, 2007

13. Chapman PB, Einhorn LH, Meyers ML, et al: Phase III multicenter randomized trial of the Dartmouth regimen versus dacarbazine in patients with metastatic melanoma. J Clin Oncol 17:2745-2751, 1999

14. Atkins MB, Lotze MT, Dutcher JP, et al: High-dose recombinant interleukin-2 therapy for patients with metastatic melanoma: Analysis of 270 patients treated between 1985 and 1993. J Clin Oncol 17:21052116, 1999

15. Algazi AP, Soon CW, Daud AI: Treatment of cutaneous melanoma: Current approaches and future prospects. Cancer Manag Res 2:197211, 2010

16. Royal RE, Steinberg SM, Krouse RS, et al: Correlates of response to interleukin-2 therapy in patients treated for metastatic renal cancer and melanoma. Cancer J Sci Am 2:91-98, 1996

17. Leach DR, Krummel MF, Allison JP: Enhancement of antitumor immunity by CTLA-4 blockade. Science 271:1734-1736, 1996

18. Hodi FS, O'Day SJ, McDermott DF, et al: Improved survival with ipilimumab in patients with metastatic melanoma. N Engl J Med 363: 711-723, 2010

19. Trinchieri G: Interleukin-12: A proinflammatory cytokine with immunoregulatory functions that bridge innate resistance and antigenspecific adaptive immunity. Annu Rev Immunol 13:251-276, 1995

20. Hendrzak JA, Brunda MJ: Interleukin-12: Biologic activity, therapeutic utility, and role in disease. Lab Invest 72:619-637, 1995

21. Andrews JV, Schoof DK, Bertagnolli MM, et al: Immunomodulatory effects of interleukin-12 on human tumor-infiltrating lymphocytes. J Immunother 14:1-10, 1993

22. Zeh HJ III, Hurd S, Storkus WJ, et al: Interleukin-12 promotes the proliferation and cytolytic activity of immune effectors: Implications for the immunotherapy of cancer. J Immunother 14:155-161, 1993

23. Mahvi DM, Henry MB, Albertini MR, et al: Intratumoral injection of IL-12 plasmid DNA-Results of a phase I/IB clinical trial. Cancer Gene Ther 14:717-723, 2007

24. Gollob JA, Mier JW, Veenstra K, et al: Phase I trial of twice-weekly intravenous interleukin 12 in patients with metastatic renal cell cancer or malignant melanoma: Ability to maintain IFN-gamma induction is associated with clinical response. Clin Cancer Res 6:1678-1692, 2000

25. Alatrash G, Hutson TE, Molto L, et al: Clinical and immunologic effects of subcutaneously administered interleukin-12 and interferon alfa-2b: Phase I trial of patients with metastatic renal cell carcinoma or malignant melanoma. J Clin Oncol 22:2891-2900, 2004 
26. Younes A, Pro B, Robertson MJ, et al: Phase II clinical trial of interleukin-12 in patients with relapsed and refractory non-Hodgkin's lymphoma and Hodgkin's disease. Clin Cancer Res 10:5432-5438, 2004

27. Daud AI, DeConti RC, Andrews S, et al: Phase I trial of interleukin-12 plasmid electroporation in patients with metastatic melanoma. J Clin Oncol 26:5896-5903, 2008http://www.ncbi.nlm.nih.gov/pmc/ articles/PMC2645111/:tool = pubmed

28. Fecher LA, Amaravadi RK, Flaherty KT: The MAPK pathway in melanoma. Curr Opin Oncol 20:183-189, 2008

29. Davies H, Bignell GR, Cox C, et al: Mutations of the BRAF gene in human cancer. Nature 417:949-954, 2002

30. Taube JM, Begum S, Shi C, et al: Benign nodal nevi frequently harbor the activating V600E BRAF mutation. Am J Surg Pathol 33:568-571, 2009

31. Chapman PB, Hauschild A, Robert C, et al: Improved survival with vemurafenib in melanoma with BRAF V600E mutation. N Engl J Med 364:2507-2516, 2011

32. McMahon M: Parsing out the complexity of RAF inhibitor resistance. Pigment Cell Melanoma Res 24:361-365, 2011

33. Adjei AA, Cohen RB, Franklin W, et al: Phase I pharmacokinetic and pharmacodynamic study of the oral, small-molecule mitogen-activated protein kinase kinase $1 / 2$ inhibitor AZD6244 (ARRY-142886) in patients with advanced cancers. J Clin Oncol 26:2139-2146, 2008

34. Dummer R, Robert C, Chapman PB, et al: AZD6244 (ARRY-142886) vs temozolomide (TMZ) in patients (pts) with advanced melanoma: An open-label, randomized, multicenter, phase II study. J Clin Oncol 26 Suppl 15, 2008. Abstract 9033

35. Patel SP, Lazar AJ, Mahoney S, et al: Clinical responses to AZD6244 (ARRY-142886)-based combination therapy stratified by gene mutations in patients with metastatic melanoma. J Clin Oncol 28 Suppl 15, 2010. Abstract 8501

36. Comparison of AZD6244 in combination with dacarbazine versus dacarbazine alone in BRAF mutation positive melanoma patientsfull text view-ClinicalTrials.gov. Available at: http://clinicaltrials.gov/ct2/show/NCT00936221. Accessed December 12, 2011

37. Docetaxel with or without AZD6244 in Melanoma (DOC-MEK)Full text view-ClinicalTrials.gov. Available at: http://clinicaltrials. gov/ct2/show/NCT01256359. Accessed December 12, 2011

38. Temsirolimus/AZD 6244 for treatment-naïve with BRAF mutant unresectable stage IV-Full text view-CalTrials.gov. Available at: http://clinicaltrials.gov/ct2/show/NCT01166126. Accessed December 12, 2011

39. Cediranib Maleate and Selumetinib in treating Patients with solid malignancies—Full text view—ClinicalTrials.gov. Available at: http:// clinicaltrials.gov/ct2/show/NCT01364051. Accessed December 12, 2011

40. Investigate Saftey, Pharmacokinetics and Pharmacodynamics of GSK2118436 and GSK1120212-Full text view—ClinicalTrials.gov. Available at: http://clinicaltrials.gov/ct2/show/NCT01072175. Accessed December 12, 2011

41. Stahl JM, Cheung M, Sharma A, et al: Loss of PTEN promotes tumor development in malignant melanoma. Cancer Res 63:2881-2890, 2003

42. Karbowniczek M, Spittle CS, Morrison T, et al: MTOR is activated in the majority of malignant melanomas. J Invest Dermatol 128:980987,2008

43. Sears R, Nuckolls F, Haura E, et al: Multiple Ras-dependent phosphorylation pathways regulate Myc protein stability. Genes Dev 14: 2501-2514, 2000

44. Reifenberger J, Wolter M, Boström J, et al: Allelic losses on chromosome arm 10q and mutation of the PTEN (MMACl) tumour suppressor gene in primary and metastatic malignant melanomas. Virchows Arch 436:487-493, 2000

45. Guldberg P, thor Straten P, Birck A, et al: Disruption of the MMACl/ PTEN gene by deletion or mutation is a frequent event in malignant melanoma. Cancer Res 57:3660-3663, 1997

46. Ernst DS, Eisenhauer E, Wainman N, et al: Phase II study of perifosine in previously untreated patients with metastatic melanoma. Invest New Drugs 23:569-575, 2005
47. Li T, Christensen SD, Frankel PH, et al: A phase II study of cell cycle inhibitor UCN-01 in patients with metastatic melanoma: A California Cancer Consortium trial. Invest New Drugs 30:741-748, 2012

48. Margolin K, Longmate J, Baratta T, et al: CCI-779 in metastatic melanoma: A phase II trial of the California Cancer Consortium. Cancer 104:1045-1048, 2005

49. Rao RD, Windschitl HE, Allred JB, et al: Phase II trial of the mTOR inhibitor everolimus (RAD-001) in metastatic melanoma. J Clin Oncol 24 Suppl 18, 2006. Abstract 8043

50. Meier FE, Beck D, Niessner K, et al: Effect of mTOR inhibitors on sorafenib-induced endoplasmic reticulum stress and apoptosis in melanoma cells. J Clin Oncol 28 Suppl. 2010. Abstract e19027

51. Maira SM, Stauffer F, Brueggen J, et al: Identification and characterization of NVP-BEZ235, a new orally available dual phosphatidylinositol 3-kinase/mammalian target of rapamycin inhibitor with potent in vivo antitumor activity. Mol Cancer Ther 7:1851-1863, 2008

52. Safety, Pharmacokinetics, and Pharmacodynamics of BEZ235 Plus MEK162 in Selected Advanced Solid Tumor Patients-Full text view-ClinicalTrials.gov. Available at: http://clinicaltrials.gov/ct2/ show/NCT01337765. Accessed December 12, 2011

53. Chabot B, Stephenson DA, Chapman VM, et al: The proto-oncogene c-kit encoding a transmembrane tyrosine kinase receptor maps to the mouse W locus. Nature 335:88-89, 1988

54. Curtin JA, Busam K, Pinkel D, et al: Somatic activation of KIT in distinct subtypes of melanoma. J Clin Oncol 24:4340-4346, 2006

55. Terada T: Low incidence of KIT gene mutations and no PDGFRA gene mutations in primary cutaneous melanoma: An immunohistochemical and molecular genetic study of Japanese cases. Int J Clin Oncol 15:453-456, 2010

56. Ugurel S, Hildenbrand R, Zimpfer A, et al: Lack of clinical efficacy of imatinib in metastatic melanoma. Br J Cancer 92:1398-1405, 2005

57. Wyman K, Atkins MB, Prieto V, et al: Multicenter phase II trial of high-dose imatinib mesylate in metastatic melanoma: Significant toxicity with no clinical efficacy. Cancer 106:2005-2011, 2006

58. Carvajal RD, Antonescu CR, Wolchok JD, et al: KIT as a therapeutic target in metastatic melanoma. Jama 305:2327-2334, 2011

59. Gentile A, Trusolino L, Comoglio PM: The met tyrosine kinase receptor in development and cancer. Cancer Metastasis Rev 27:85-94, 2008

60. Hsu MY, Meier F, Herlyn M: Melanoma development and progression: A conspiracy between tumor and host. Differentiation 70:522536, 2002

61. Puri N, Ahmed S, Janamanchi V, et al: c-Met is a potentially new therapeutic target for treatment of human melanoma. Clin Cancer Res 13:2246-2253, 2007

62. Economou MA, All-Ericsson C, Bykov V, et al: Receptors for the liver synthesized growth factors IGF-1 and HGF/SF in uveal melanoma: Intercorrelation and prognostic implications. Acta Ophthalmol 86 Thesis 4:20-25, 2008

63. Gordon MS, Vogelzang NJ, Schoffski P, et al: Activity of cabozantinib (XL184) in soft tissue and bone: Results of a phase II randomized discontinuation trial (RDT) in patients (pts) with advanced solid tumors. J Clin Oncol 29 Suppl. 2011. Abstract 3010

64. Study of XL184 in adults with advanced malignancies.Clinical Trials.gov. Available at: http://www.clinicaltrials.gov/ct2/show/study/ NCT00940225. Accessed December 12, 2011

65. Eder JP, Appleman L, Heath E, et al: A phase I study of a novel spectrum selective kinase inhibitor (SSKI), XL880, administered orally in patients (pts) with advanced solid tumors (STs). J Clin Oncol 24 Suppl 18, 2006. Abstract 3041

66. Yarden Y: The EGFR family and its ligands in human cancer: Signalling mechanisms and therapeutic opportunities. Eur J Cancer 37 Suppl 4:S3-S8, 2001

67. Sparrow LE, Heenan PJ: Differential expression of epidermal growth factor receptor in melanocytic tumours demonstrated by immunohistochemistry and mRNA in situ hybridization. Australas J Dermatol 40:19-24, 1999

68. Trent JM, Meyskens FL, Salmon SE, et al: Relation of cytogenetic abnormalities and clinical outcome in metastatic melanoma [Pub- 
lished correction appears in N Engl J Med 323:1283, 1990]. N Engl J Med 322:1508-1511, 1990

69. Rákosy Z, Vízkeleti L, Ecsedi S, et al: EGFR gene copy number alterations in primary cutaneous malignant melanomas are associated with poor prognosis. Int J Cancer 121:1729-1737, 2007

70. Ueno Y, Sakurai H, Tsunoda S, et al: Heregulin-induced activation of ErbB3 by EGFR tyrosine kinase activity promotes tumor growth and metastasis in melanoma cells. Int J Cancer 123:340-347, 2008

71. Djerf EA, Trinks C, Abdiu A, et al: ErbB receptor tyrosine kinases contribute to proliferation of malignant melanoma cells: Inhibition by gefitinib (ZD 1839). Melanoma Res 19:156-166, 2009

72. Buac K, Xu M, Cronin J, et al: NRGl/ERBB3 signaling in melanocyte development and melanoma: Inhibition of differentiation and promotion of proliferation. Pigment cell. Melanoma Res 22:773-784, 2009

73. Wellbrock C, Gómez A, Schartl M: Signal transduction by the oncogenic receptor tyrosine kinase Xmrk in melanoma formation of Xiphophorus. Pigment Cell Res 10:34-40, 1997

74. Prickett TD, Agrawal NS, Wei X, et al: Analysis of the tyrosine kinome in melanoma reveals recurrent mutations in ERBB4. Nat Genet 41: 1127-1132, 2009

75. Wyman K, Kelley M, Puzanov I, et al: Phase II study of erlotinib given daily for patients with metastatic melanoma (MM). J Clin Oncol 24 Suppl 18, 2006. Abstract 18002

76. Patel S, Bedikian A, Kim K, et al: A phase II study of gefitinib in patients with metastatic melanoma. J Clin Oncol 27 Suppl 15, 2009. Abstract 9057

77. Wyman K, Spigel D, Puzanov I, et al: A multicenter phase II study of erlotinib and bevacizumab in patients with metastatic melanoma. J Clin Oncol 25 Suppl 18, 2007. Abstract 8539

78. Grignol VP, Olencki T, Relekar K, et al: A phase 2 trial of bevacizumab and high-dose interferon alpha $2 \mathrm{~b}$ in metastatic melanoma. J Immunother 34:509-515, 2011

79. Hodi FS, Friedlander PA, Atkins MB, et al: A phase I trial of ipilimumab plus bevacizumab in patients with unresectable stage III or stage IV melanoma. J Clin Oncol 29 Suppl. 2011. Abstract 8511

80. von Moos R, Seifert B, Simcock M, et al: First-line temozolomide combined with bevacizumab in metastatic melanoma: A multicenter phase II trial (SAKK 50/07). Ann Oncol 23:531-536, 2012

81. Boasberg PD, Weber RW, Cruickshank S, et al: Phase II trial of nabpaclitaxel and bevacizumab as first-line therapy in patients with unresectable melanoma. J Clin Oncol 29 Suppl. 2011. Abstract 8543

82. Perez DG, Suman V, Amatruda T, et al: Phase II trial of carboplatin, weekly paclitaxel, and biweekly bevacizumab in patients with unresectable stage IV melanoma. J Clin Oncol 25 Suppl 18, 2007. Abstract 8560

83. Kottschade LA, Suman V, Perez DG, et al: A randomized phase II trial of temozolomide (TMZ) and bevacizumab (BEV) or nab-paclitaxel (nab-P)/carboplatin (CBDCA) and bevacizumab (BEV) in patients with unresectable stage IV metastatic melanoma: A north central cancer treatment group study (N0775). J Clin Oncol 29 Suppl. 2011. Abstract 8532

84. di Pietro A, Ferrucci P, Munzone E, et al: Dacarbazine (DTIC) plus bevacizumab (B) combination therapy in chemotherapy (CTh)-naïve advanced melanoma (MM) patients (pts): A phase II study. J Clin Oncol 28 Suppl 15, 2010. Abstract 8536

85. Hainsworth JD, Infante JR, Spigel DR, et al: Bevacizumab and everolimus in the treatment of patients with metastatic melanoma: A phase 2 trial of the Sarah Cannon Oncology Research Consortium. Cancer $116: 4122-4129,2010$

86. Si L, Chi Z, Yuan X, et al: Durable response of the triple combination of temozolomide, sorafenib, and bevacizumab to treat refractory stage IV acral melanoma. J Clin Oncol 28 Suppl 15, 2010. Abstract 8564

87. McClay EF, Bessudo A, Frakes L, et al: A phase I/II trial of the combination of bevacizumab, oxaliplatin, and sorafenib in patients with metastatic melanoma. J Clin Oncol 26, 2008. Abstract 20020
88. Bauknecht T, Gross G, Hagedorn M: Epidermal growth factor receptors in different skin tumors. Dermatologica 171:16-20, 1985

89. Liu B, Zhang H, Li S, et al: The expression of c-erbB-1 and c-erbB-2 oncogenes in basal cell carcinoma and squamous cell carcinoma of skin. Chin Med Sci J 11:106-109, 1996

90. Brabender J, Danenberg KD, Metzger R, et al: Epidermal growth factor receptor and HER2-neu mRNA expression in non-small cell lung cancer is correlated with survival. Clin Cancer Res 7:1850-1855, 2001

91. Shimizu T, Izumi H, Oga A, et al: Epidermal growth factor receptor overexpression and genetic aberrations in metastatic squamous cell carcinoma of the skin. Dermatology 202:203-206, 2001

92. Gingras AC, Kennedy SG, O'Leary MA, et al: 4E-B1, a repressor of mRNA translation, is phosphorylated and inactivated by the Akt (PKB) signaling pathway. Genes Dev 12:502-513, 1998

93. Perrotte $\mathrm{P}$, Matsumoto $\mathrm{T}$, Inoue $\mathrm{K}$, et al: Anti-epidermal growth factor receptor antibody $\mathrm{C} 225$ inhibits angiogenesis in human transitional cell carcinoma growing orthotopically in nude mice. Clin Cancer Res 5:257-265, 1999

94. Suen JK, Bressler L, Shord SS, et al: Cutaneous squamous cell carcinoma responding serially to single-agent cetuximab. Anti Cancer Drugs 18:827-829, 2007

95. Vano-Galvan S, Rios-Buceta L, Ma DL, et al: Cetuximab induced hypertrichosis of the scalp and eyelashes. J Am Acad Dermatol 62:531532,2010

96. Arnold AW, Bruckner-Tuderman L, Zuger C, et al: Cetuximab therapy of metastasizing cutaneous squamous cell carcinoma in a patient with severe recessive dystrophic epidermolysis bullosa. Dermatology 219: 80-83, 2009

97. Thienelt CD, Bunn PA Jr, Hanna N, et al: Multicentered phase I/II study of cetuximab in combination with paclitaxel and carboplatin in untreated patients with stage IV non-small cell lung cancer. J Clin Oncol 23:8786-8793, 2005

98. Vega-Villegas E, Awada R, Mesia L, et al: A phase I study of cetuximab in combination with cisplatin or carboplatin and 5-FU in patients with recurrent or metastatic squamous cell carcinoma of the head and neck. Proc Am Assoc Cancer Res 22:2020, 2003

99. Herbst RS, Arquette M, Shin DM, et al: Phase II multicenter study of the epidermal growth factor receptor antibody cetuximab and cisplatin for recurrent and refractory squamous cell carcinoma of the head and neck. J Clin Oncol 23:5578-5587, 2005

100. Benvenuti S, Sartore-Bianchi A, Di Nicolantonio F, et al: Oncogenic activation of the RAS/RAF signaling pathway impairs the response of metastasis colorectal cancers to anti-epidermal growth factor receptor antibody therapies. Cancer Res 67:2643-2648, 2007

101. Di Nicolantonio F, Martini M, Molinari F, et al: Wild-type BRAF is required for response to panitumumab or cetuximab in metastatic colorectal cancer. J Clin Oncol 26:5705-5712, 2008

102. Vanhoefer U, Tewes M, Rojo F, et al: Phase I study of the humanized antiepidermal growth factor receptor monoclonal antibody EMD 72000 in patients with advanced solid tumors that express the EGFR. J Clin Oncol 22:175-184, 2004

103. Seiden MV, Burris HA, Matulonis U, et al: A phase II trial of EMD 72000 (matuzumab), a humanized anti-EGFR monoclonal antibody in patients with platinum-resistance ovarian and primary peritoneal malignancies. Gynecol Oncol 104:727-731, 2007

104. Ciardiello F, Caputo R, Bianco R, et al: Antitumor effect of potentiation of cytotoxic drugs activity in human cancer cells by ZD-1839 (Iressa) an epidermal growth factor receptor selective tyrosine kinase inhibitor. Clin Cancer Res 6:2053-2063, 2000

105. Glisson BS, Kim ES, Kies MS, et al: Phase II study of gefitinib in patients with metastatic/recurrent squamous cell carcinoma of the skin. J Clin Oncol 24 Suppl 18, 2006. Abstract 5531

106. Moyer JD, Barbacci EG, Iwata KK, et al: Induction of apoptosis and cell cycle arrest by CP-358,774, an inhibitor of epidermal growth factor receptor tyrosine kinase. Cancer Res 57:4838-4848, 1997

107. Akita RW, Sliwkowski MX: Preclinical studies with erlotinib (Tarceva). Semin Oncol 30:15-24, 2003 